\title{
MeerTRAP: A pulsar and fast transients survey with MeerKAT
}

\author{
S. Sanidas, M. Caleb, L. Driessen, V. Morello, K. Rajwade and \\ B. W. Stappers ${ }^{\dagger}$
}

Jodrell Bank Centre for Astrophysics, The University of Manchester, Alan Turing Building, Manchester, M13 9PL, United Kingdom

email: sotirios.sanidas@manchester.ac.uk

\begin{abstract}
We present a brief overview of MeerTRAP, a real-time, fully commensal survey for pulsars and fast transients with the MeerKAT radio telescope in South Africa. MeerTRAP will combine the excellent sensitivity of MeerKAT with an unprecedented amount of on-sky time in order to significantly extend the parameter space covered by similar, previous or ongoing, surveys with other radio telescopes. Here, we will give a brief overview of the project.
\end{abstract}

Keywords. pulsars: general, instrumentation: interferometers

\section{Introduction}

In the 50 years since the discovery of the first pulsar, numerous surveys have been undertaken by all major radio telescopes in the world aiming to probe their galactic population. The result was to unveil the even richer observational manifestations of neutron stars through the discovery of interesting binaries, intermittent/nulling pulsars and Rotating RAdio Transients (RRATs), suggesting that a key characteristic for a successful survey of all classes of radio emitting neutron stars is not only a sensitive instrument, but also extensive temporal sky coverage. This last characteristic is also crucial for finding new Fast Radio Bursts (FRBs) (Lorimer et al. (2007)), ms-duration extremely bright radio pulses, with only one of them established to repeat, and having a confirmed localisation (Spitler et al. (2016), Marcote et al. (2017), Tendulkar et al. (2017)). MeerTRAP, more (meer) TRAnsients and Pulsars, is a survey for pulsars and fast transients with MeerKAT, that aims to address all these requirements, providing extensive sky coverage, excellent sensitivity and accurate localisation.

\section{MeerTRAP Overview}

MeerTRAP will be a fully commensal survey of the Southern Sky with the MeerKAT radio telescope. It will perform observations simultaneously with all the MeerKAT Large Survey Projects (MLSPs), granting it an on-sky time of many thousands of hours. Moreover, the various observing strategies of the MLSPs, will allow for multiple revisits of the same areas in the sky, enabling the exploration of an extremely wide range of variability timescales for the discovery of radio transients and pulsars (Fig. 1).

The MeerTRAP simultaneous observations will use two different modes: coherent and incoherent. In the coherent mode, MeerTRAP will use the 40 innermost dishes of MeerKAT in order to create 400 beams $^{\ddagger}$ on the sky with a total Field of View (FoV)

${ }^{\dagger}$ This project has received funding from the European Research Council (ERC) under the European Union's Horizon 2020 research and innovation programme (grant agreement $\mathrm{n}^{\circ}$ 694745).

${ }^{\ddagger}$ The development of the beamformer is led by MPIfR. 


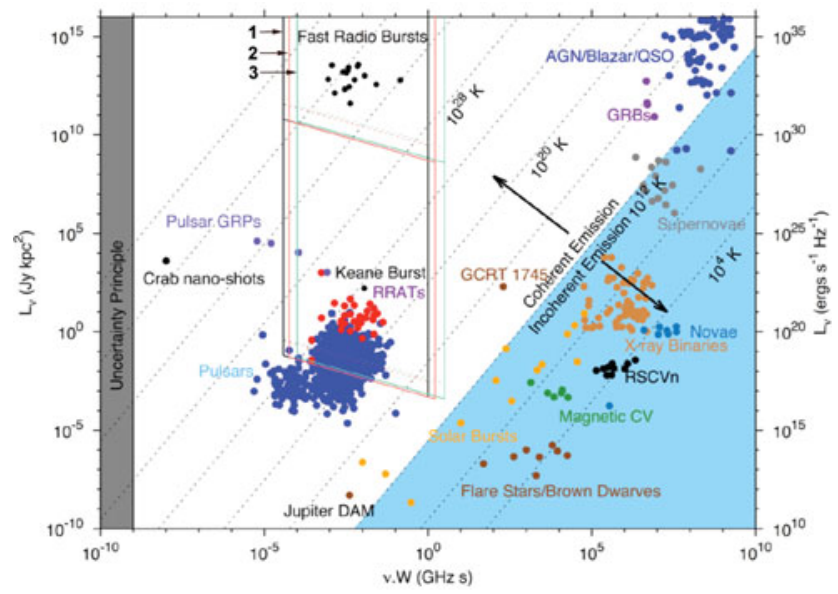

Figure 1. Transient parameter space showing specific luminosity against the product of observing frequency and transient duration. The boxes correspond to the $1-\sigma$ sensitivity curves for MeerTRAP for source distances of $1 \mathrm{kpc}$ (lower curves) and $1 \mathrm{Gpc}$ (upper curves) and coherent (solid) and incoherent (dashed) modes. The left and right edges are determined by the range of observing frequencies and the widths of pulses that are searched for, i.e. from about $1 \mathrm{~ms}$ to $1 \mathrm{~s}$. The different colours correspond to the different frequency bands: UHF (black, line 1), L-band (salmon, line 2) and S-band (cyan, line 3). One can see that all the known FRBs could be easily detected with MeerTRAP. Most pulsars would be detected via their single pulses as would all RRATS. Credit: Keane (after Cordes)

$\sim 0.1^{\circ}$, providing an initial localisation for the discoveries of ten arcseconds or less (depending on $\mathrm{S} / \mathrm{N}$ ). In the incoherent mode, the intensities of all 64 dishes will be added in order to create a less sensitive, but much wider beam on the sky with FoV $\sim 1.27^{\circ}$. The data streams from both the coherent and incoherent beams will be ingested to the MeerTRAP GPU cluster, and searched in real time for pulsars and fast transients. In this way, MeerTRAP is going to probe simultaneously the population of faint, and more common transients, and the population of bright, but rarer ones.

MeerTRAP will also be utilising transient buffers, so when an interesting signal is detected the data from all dishes will be stored on disk. This will allow us to perform imaging with all 64 dishes and increase our sensitivity by $50 \%$ for coherent beam detections, and by a factor of 8 for incoherent beam detections. Moreover, the longest baselines available will be used in order to provide even better localisation of our discoveries, or even re-beamform on the refined position to increase the detection significance.

All MeerTRAP observations will also be shadowed by MeerLICHT, a 64-cm fully robotic optical telescope which is twinned with MeerKAT, offering the world's first continuous simultaneous radio-optical telescope combination. MeerTRAP will have access to all MeerLICHT data, allowing us to look for prompt or afterglow emission from our discoveries, as well as looking for any possible optical emission preceding the radio signals. Such access to multi-wavelength data will further increase our localisation capabilities, and enable multiwavelength studies of bursts and host galaxies.

\section{References}

Lorimer, D. R. et al. 2007, Science, 318, 5851, 777-780

Spitler, L. G. et al. 2016, Nature, 531, 202-205

Marcote, B. et al. 2017, ApJ (Letters), 834, 2

Tendulkar, S. P. et al. 2017, ApJ (Letters), 834, 2 